\title{
Global Citizenship Education - eine pädagogische Antwort auf die „große Regression“?
}

\begin{abstract}
Zusammenfassung
Dieser Beitrag möchte nicht in den Chor derer einstimmen, die den rechten Nationalismus als die „große Regression“ beklagen und dem gegenüber die liberale Demokratie als ideale Realisierung der europäischen Aufklärung hochhalten. Er postuliert vielmehr, dass die Problematik einer demokratischen Bildung heute darin besteht, zwischen der Skylla eines „regressiven“ Nationalismus und der Charybdis einer neoliberalen „Weltoffenheit“ zu navigieren. Weltbürgerlichkeit ist nicht per se ein Gegenkonzept zu Nationalismus. Wie postkoloniale Theoretiker/-innen gezeigt haben, ist vielmehr die europäische Weltbürgerlichkeit ein begleitendes und ideologisierendes Element der kolonialen Unterwerfung des Globalen Südens. Dieser Beitrag argumentiert für eine Bildung zur Demokratie aus dekolonial-kosmopolitischer Perspektive.
\end{abstract}

Schlüsselworte: methodischer Nationalismus, dekolonialer Kosmopolitismus, neuer Nationalismus, Neoliberalismus

\begin{abstract}
This paper refuses the idea that (neo-)liberal democracy is the only alternative to the "great regression", the rise of right-wing nationalism. Education for democracy, I argue instead, has to criticize both, the open racism of the "new nationalism" as well as the "cosmopolitan" liberalism that is at the origin of business globalization and a growing divide between haves and have-nots. In order to help building democracy at a worldwide scale, a truly democratic education has to adopt a decolonial global citizenship perspective, as a concrete utopia.
\end{abstract}

Keywords: methodological nationalism, decolonial cosmopolitanism, new nationalism, neoliberalism

\section{Was der Gelbwesten-Protest zeigt}

Bei den Protesten der so genannten gilets jaunes (Gelbwesten) gegen die Maßnahmen der Regierung Macron hat ein Slogan Furore gemacht: Ende der Welt oder Ende des Monats? Wie unterschiedlich aber die Erzählungen zu diesem Slogan ausfallen, ist bezeichnend für die Auseinandersetzung zwischen Neoliberalismus und Nationalismus, weit über Frankreich hinaus. Laut der einen Version hätten sich die Gelbwesten beklagt, dass die neue ,Ökosteuer' auf Benzin und Diesel - mit der Sorge um den Klimawandel, also um das ,Ende der Welt', begründet - den einfachen Menschen das ,Ende des Monats'schwermache, weil sie finanziell nicht durchkämen. Es ist die Erzählung von der aufgeklärten Elite gegen die arme, aber auch engstirnig und letztlich nationalistisch denkende Masse, die nicht zu Opfern für das große Ganze bereit ist.

In der alternativen Version wird hingegen betont, dass die sozial Schwachen als einzige zur Kasse gebeten würden. Der Treibstoff für Flugzeuge werde zum Beispiel nicht besteuert. Und außerdem sei unter den Forderungen der Gelbwesten sehr wohl viel Ökologisches zu finden, wie etwa das Verbot von Glyphosat, das die Regierung gerade für drei weitere Jahre zugelassen hat. Somit drücke der Slogan nur die Verachtung der reichen Elite - die auch am meisten für Umweltverschmutzung und Klimawandel verantwortlich sei - gegenüber der Masse der Bevölkerung aus. In dieser Version wird das ausgesprochen, was die Proteste in Frankreich insgesamt zeigen: Es geht um das Scheitern des neoliberalen Projekts, das sich als ,fortschrittlich', ,kosmopolitisch' und ,modern verkauft oder zumindest beansprucht, das kleinere Übel im Vergleich zu einem aggressiven Nationalismus zu sein, wie ihn in Frankreich Marine Le Pen verkörpert. Die Gelbwesten haben den französischen Präsidenten zur Rücknahme einer ganzen Reihe von Maßnahmen gezwungen und ihr Erfolg ist - bei aller Problematik dieser neuen Bewegung - quasi ein materieller Beweis für die Notwendigkeit und (im Keim) auch der Existenz eines dritten Weges. ${ }^{1}$

Die Gelbwesten attackierten nicht nur die Politik der Regierung Macron, sondern auch ihre Ideologie: Diese macht die Gefahr einer nationalistischen und teilweise totalitären Regression - oft verstanden als Reaktion auf die Zunahme von Migrationsströmen nach Europa bzw. in andere Länder des Globalen Nordens - als Hauptproblem aus und betrachtet dem gegenüber alles, was über das Nationale hinausweist, wie die EU oder die Globalisierung insgesamt, als „fortschrittlich“, wenn auch die „Schattenseiten“ der Globalisierung und die „Fehler" der Union bedauert werden. Die Gelbwesten wiesen diese moralische Erpressung zurück und wandten sich sowohl gegen das neoliberal-kosmopolitische als auch gegen das mit sozialer Demagogie operierende na- 
tionalistische Projekt. Sie wollten sich für keine dieser beiden Alternativen entscheiden und haben auch deren Vertretungen (Gewerkschaften und Parteien) abgelehnt.

\section{Bildung und Erziehung für Demokratie}

Für unser Anliegen, Bildung für Demokratie, bedeutet das einen Anstoß, die Frage nach der Erziehung zur Demokratie neu zu stellen. Denn Demokratieerziehung als simples Einschwören auf den Status quo der liberalen Demokratie ist inzwischen weder sachlich angemessen noch pädagogisch aussichtsreich. Vielmehr müssen wir uns mit zwei Fragen beschäftigen:

- Was ist an der "großen Regression“ so faszinierend, dass ein guter Teil der Bevölkerungen - und natürlich auch der Lernenden - in Europa oder den USA solche Bewegungen unterstützt?

- Was ist an der Vision der globalen Weltgemeinschaft, wie sie vom demokratischen neoliberalen Kapitalismus realisiert wird, so abstoßend, dass sie ein guter Teil eben dieser Bevölkerungen - und Lernenden - ablehnt?

Um diese Fragen zu beantworten, müssen wir den methodischen Nationalismus zurückweisen, der die „große Regression“, das Zurückfallen hinter einen als unhintergehbar geglaubten Grad an Zivilisierung, als ein letztlich unerklärliches Phänomen der Renationalisierung sieht, und vielleicht noch als bedauerliche Reaktion auf die Zunahme von Migration, Terrorismus und importierten anti-demokratischen Ideologien.

Dagegen ist zunächst festzuhalten, worauf der Protest der Gelbwesten aufmerksam gemacht hat: Es ist die wirtschaftliche Globalisierung, von der gerade im Westen eine Elite enorm profitiert, die zugleich neue Armut und neue Abhängigkeiten in den Industriestaaten selbst schafft. Darüber hinaus haben die wirtschaftliche Expansion sowie militärische Interventionen zu ihrem Schutz zur wirtschaftlichen und politischen Destabilisierung vieler Regionen des globalen Südens geführt. Deswegen ist auch jenes Narrativ nur die halbe Wahrheit, dass es um die Verteidigung der europäischen Aufklärung gegen den „mittelalterlichen“ Islam der Migrant/-innen gehe - eine Erzählung die gerade in Frankreich nach den islamistischen Attentaten 2015 so glaubwürdig wirkte. „Im unmittelbaren Zusammenhang mit Terrorismus und Migration steht die Tatsache, dass sich rund um den Globus die Gebiete ausdehnen, in denen es keine Staatlichkeit mehr gibt" (Geiselberger, 2017, S. 7). Vieles, was wir nur aus dem Blickwinkel unserer Nationalstaaten betrachten, hängt eben in Wirklichkeit mit globalen Veränderungen zusammen, zu denen wir beigetragen haben, und die nun massiv auf uns rückwirken.

Hinzu kommt ein Phänomen, das Bewegungen wie die Gelbwesten keineswegs auf ihrem Radar hatten. Dieses prägt gleichwohl wesentlich die Gesamtsituation: Es gibt einen Widerstand aus dem globalen Süden gegen die westliche Globalisierung. Das zeigt sich etwa im wesentlich selbstbewussteren Auftreten vieler Staaten des globalen Südens auf dem internationalen Parkett, die nun versuchen, einer neokolonialen Politik die Stirn zu bieten; im Anwachsen sozialer Widerstandsbewegungen, von denen wir meist nur die terroristische Seite wahrnehmen; und schließlich wohl erstmals auch in der postkolonialen Herausforderung nicht nur westlicher Politik, sondern auch von westlicher Kultur, Wissenschaft und Weltbildern, die unsere bisherigen Denkschemata und Erklärungsmuster grundsätzlich infrage stellen.

\section{„Vom Regen des progressiven Neoliberalismus in die Traufe des reaktionären Populismus"}

Somit kommen wir zu einer Neubewertung der Situation und zu einer Infragestellung beider Narrative, sowohl des „Regens des progressiven Neoliberalismus“ als auch der "Traufe des reaktionären Populismus" (Fraser, 2017). Wir können in den ,reaktionären Haltungen` der Bevölkerung im Westen, die zur Wahl von Donald Trump, dem Brexit und dem Aufstieg rechts-nationalistischer Parteien wie AfD oder FPÖ geführt haben, eine Form der Politisierung und damit der Dynamisierung unserer Gesellschaften sehen. Das Politische entsteht, wenn diejenigen, denen man politische Äußerungen verweigert oder nicht zutraut, das Wort ergreifen, und wenn Themen, die nicht als politisch geeignet gelten, aufs Tapet gebracht werden: „Vom demos ist derjenige, der spricht, obwohl er nicht zu sprechen hat, derjenige, der an etwas teilnimmt, woran er keinen Anteil hat" (Rancière, 2008, S. 21). Diese Politisierung mag subjektiv als demokratischer Akt verstanden werden, als Aufschrei derer, die bislang von der politischen Klasse nicht gehört wurden. Es ist freilich ein Streben nach sozialer Verbesserung und persönlicher Würde, allerdings gepaart mit nationalistischer Abschottung und rassistischer Abwehr aller „Anderen“. Wir haben es mit einer „Rebellion der Armen [zu tun, W. W.], die sich sozusagen im Gegner geirrt hatten", wie Paul Mason (2017, S. 163) mit Bezug auf den britischen Brexit schreibt. Wenn wir den Nationalismus und Rassismus dieser Bewegungen zurückweisen, müssen wir doch gleichzeitig ihren Widerstand gegen sozialen Abstieg und für Anerkennung und Würde als berechtigt anerkennen. Dabei kommt es darauf an, diese Verbindung vom Kampf um eigene Würde und rassistische Verweigerung der Würde der anderen aufzulösen.

Aber auch die scheinbare Alternative, also der ,demokratische ' Universalismus und Globalismus der westlichen Eliten, ebenso wie ihre bedingungslose Treue zur ,Europaidee ${ }^{\text {- }}$ - ohne kritische Prüfung der konkreten EU-Politik - ist zu hinterfragen. Wie ausgeführt, hat die herrschende Politik die Schere zwischen Arm und Reich in skandalöser Weise aufgetan und damit erst dem ,neuen Nationalismus' als Gegenbewegung eine Chance gegeben. Und ihr Universalismus ist, wie postkoloniale Kritiker (z. B. Mignolo, 2011) gezeigt haben, nichts anderes als die partikulare Form, wie die Eliten ihre eigenen Interessen verteidigen, indem sie sie als allgemeine Interessen ausgeben. Hier ist wieder das Beispiel Frankreich aufschlussreich. Denn in diesem Musterland der universalen Menschenrechte richtet sich die heutige Politik nicht nur gegen breite Teile der eigenen Bevölkerung, sondern arbeitet auch auf globaler Ebene an der Durchsetzung ,nationalistischer Ziele. Es geht um einschränkende Handelsverträge mit afrikanischen Staaten bis hin zu Militärinterventionen zur Sicherung von Rohstoffen - etwa Uran für die Atomindustrie und die Atomwaffen der ,force de frappe'. Dies alles wird mit Formulierungen wie ,Partnerschaft', ,Hilfe zur Entwicklung' oder ,gemeinsamer Kampf gegen Terrorismus‘ ideologisch bemäntelt. Demokratie und kritisches Weltbürgertum bleiben auf der Strecke. Vielmehr scheint sich folgendes Verdikt zu bestätigen: „Cosmopolitanism is a concern of Western scholarship“ (Mignolo, 2011, S. 252). Wenn wir den demokratischen und weltbürgerlichen Anspruch, den die neoliberalen Eliten auf ihre Fahnen schreiben, vor ihnen selbst retten wollen, müssen wir ihn sozialkritisch und dekolonial de- und rekonstruieren. Mignolo tritt in diesem Sinne für einen 
„decolonial cosmopolitanism“ ein. Er argumentiert, dass das Ergebnis „cosmopolitan localisms” sein werden. Vom Universalismus kann zwar das Bewusstsein von der Welteinheit kritisch übernommen werden, nicht aber die Vorgangsweise, westliche als universale Lösungen anzubieten: „Decolonial cosmopolitanism is not one more among others, but a project that promotes the shift in the terms of the conversation: from imperial cosmopolitanism (or cosmopolitanism without modifiers and without parenthesis) to cosmopolitan localisms (coexistence of cosmopolitanism with modifiers and with parenthesis)“ (Mignolo, 2011, S. 293).

\section{GCED - auf dem Weg zu einer „dekolonialen" Politischen Bildung}

Bildung zur Demokratie muss somit die Frage einschließen, welche Folgen die Existenz der westlichen Demokratien und ihrer kosmopolitischen Praxen nach sich zieht. Das bedeutet auch ein Thematisieren von Kolonialismus und Neokolonialismus: „Cosmopolitanism shall be thought out in relation to a heterogenous historico-structural conception of history and society [...] and world order, rather than in a unilinear narrative of history and a hierarchical organization of society. Decolonial cosmopolitanism shall be 'the becoming of a pluriversal world order' built on and dwelling in the global borders of modernity/coloniality " (Mignolo, 2011, S. 270).

Bildung zur Demokratie kann nur gelingen, so meine These, wenn sie über das nationale Paradigma hinausgedacht wird. Das bedeutet keineswegs, dass die nationalstaatlich verfasste Demokratie - die einzige, die wir bislang haben - zu missachten oder als obsolet zu betrachten wäre. Es geht vielmehr darum, das Lokale wie das Nationale im Paradigma des dekolonialen Kosmopolitismus wahrzunehmen.

Global Citizenship Education (GCED) ist die pädagogische Umsetzung dieses Konzepts. GCED ist diesem Verständnis nach nicht einfach eine besondere Aufmerksamkeitsrichtung oder ein Spezialgebiet Politischer Bildung, sondern ein neues Paradigma, das eine andere Wahrnehmung der Ziele und Aufgaben Politischer Bildung zur Folge hat. Das Entscheidende ist dabei nicht die ,globale Weltsicht'. Denn ein Bezug zum Globalen ist heute wohl bei nahezu allen pädagogischen Konzepten eine Selbstverständlichkeit (z. B. Sander, 2018). Es ist auch nicht der manchmal erhobene Anspruch, für eine, weltbürgerliche Identität‘ auszubilden. Es bedarf zwar tatsächlich eines Denkens in weltbürgerlichen Kategorien, doch bleibt die eigene Weltsicht notwendigerweise immer vom jeweiligen Standpunkt geprägt: "Your location in the colonial matrix of power shapes the way you look at the world“ (Mignolo, 2011, S. 293). Insofern könnte man von Identität als global citizen immer nur im Plural und als multiple Identität sprechen. Hingegen als singuläre neue Identität verstanden wäre, weltbürgerliche Identität' wiederum ein universalistisch-imperiales Konzept. Was das Spezifische von GCED ausmacht, ist also nicht der Bezug zum Globalen, sondern vielmehr die normative Ausrichtung auf globale Gerechtigkeit, die auch eine kritische, dekoloniale Überprüfung westlicher Bildungskonzepte selbst beinhaltet. Was die Politische Bildung angeht, müssen wir uns auch mit einer „pluriversal world order of democracies“ um Mignolo (2011, S. 270) zu paraphrasieren.

GCED bietet die Chance einer politischen Bildung, die die Idee der demokratischen Partizipation ernst nimmt und damit unweigerlich auch auf wesentliche Defizite der Demokratie auf- merksam macht. Sie legt damit die Lernenden nicht von vornherein auf die Verteidigung einer limitierten Demokratie als der Demokratie schlechthin fest. Nun ist die Erkenntnis der Unvollkommenheit jeder Demokratie klassisches politikwissenschaftliches Wissen. Spätestens seit Norberto Bobbios (1985) Essay Il futuro della democrazia gelten „die nicht eingehaltenen Versprechen der Demokratie" (ebd., S. 25) als wissenschaftlich etablierte Tatsache, ebenso wie die strukturellen Hindernisse, die er diesbezüglich identifiziert. Und in diesem Sinne ist auch „Demokratie lernen als Kritik der Demokratie“ (Henkenborg, 2009, S. 287) ein anerkannter Grundsatz Politischer Bildung.

Aber all diese Kritiken verbleiben im nationalen Paradigma und in einer Politikwissenschaft, die die Geschichte der westlich-europäischen Demokratie als die Geschichte der Demokratie schlechthin erzählt. Bereits die Repräsentanten der ersten Welle des Postkolonialismus, wie Frantz Fanon oder Aimé Césaire, haben auf die doppelten Standards der westlichen Zivilisation verwiesen: Demokratie nach innen, Imperialismus nach außen. Wie Césaire zeigt, war das, was der Europäer „Hitler nicht verzeiht, nicht das Verbrechen an sich, das Verbrechen am Menschen, daß es nicht die Erniedrigung des Menschen an sich, sondern daß es das Verbrechen gegen den weißen Menschen ist, daß es die Demütigung des Weißen ist und die Anwendung kolonisatorischer Praktiken auf Europa, denen bisher nur die Araber Algeriens, die Kulis in Indien und die Neger Afrikas ausgesetzt waren" (Césaire, 1968, S.12, H.i.O.).

Wenn die „didaktische Grundfrage“ Politischer Bildung lautet: „Wie können Menschen und Gruppen in der Gesellschaft lernen, die Probleme und Konflikte in ihrem Zusammenleben durch die Herstellung und Durchsetzung von allgemeiner Verbindlichkeit demokratisch zu gestalten und zu regeln, und welche Probleme und Konflikte müssen sie dabei wiederum bewältigen?“ (Henkenborg, 2009, S. 284), dann muss diese Innenperspektive unbedingt auch durch eine Außenperspektive ergänzt werden. Konsequenterweise wären dann zwei Fragen zu stellen: Wer gehört zu ,der Gesellschaft' und wer ist ausgeschlossen? Sowie: Wie kann gelernt werden, auch die Beziehungen zwischen Gesellschaften demokratisch zu gestalten und deren Konflikte demokratisch zu regeln?

GCED - mit ihrem Anspruch, dass Demokratie weltweit gelten muss, und dass es vielfältige Formen von Demokratie geben kann - geht diesen entscheidenden Schritt weiter. Sie macht den Blick frei für die Defizite bestehender Demokratien, die aus einer dekolonialen Perspektive bestehen, und stellt damit erst wirklich die Frage der Demokratie in ihrem vollen Umfang. GCED vertritt die Ambition auf eine weltweite demokratische Ordnung, ohne dafür ein bestimmtes Modell vorzuschlagen. Es ist ein „Kosmopolitismus ohne Illusionen“ (Benhabib, 2016). Dabei sind die zwei genannten Aspekte nicht nur theoretisch, sondern auch für die Praxis des Demokratielernens besonders relevant:

- Von einem dekolonial aufgeklärten kosmopolitischen Standpunkt ist die Tatsache, dass es in den europäischen Einwanderungsstaaten zwei Klassen von Menschen gibt - solche mit allen staatspolitischen Rechten und solche, denen diese (und zunehmend auch soziale Rechte) verweigert werden -, keine humanitär bedauerliche Angelegenheit, sondern ein schwerwiegendes demokratiepolitisches Defizit. Wenn ein beachtlicher Teil der Wohnbevölkerung in die demokratischen 
Entscheidungen nicht einbezogen wird, bedeutet dies eine Spaltung der Gesellschaft. Diese kann aber nicht einfach aufgehoben werden, denn der souveräne Nationalstaat beruht eben auf dem Prinzip der Staatsbürgerschaft und damit der Unterscheidung zwischen denen, die politische Rechte haben, und denen, die sie nicht haben. Hier steht ein demokratiepolitisches Dilemma an, das umso größer wird, je höher durch Flucht und Migration der Anteil an Menschen ohne volle Rechte wird. Diese Ungleichbehandlung der Migrant/inn/en beruht, wie die postkoloniale Theorie gezeigt hat, letztlich auf (neo)kolonialen Praktiken, die das rassistische Muster der Unterscheidung entwickelt und perfektioniert hat, wonach es solche gibt, denen Rechte zustehen, und solche, denen sie nicht zustehen. Es handelt sich sozusagen um die Anwendung des Kolonialismus, nach innen': „Citizenship seems like a new line of stratification: voting rights, health benefits, jobs - so much depends on it. But this is the same line of stratification that constituted inequality within the colonial empires. Citizenship today is merely the hierarchy of empire reinscribed onto the structures of an ostensibly postimperial world“ (Go, 2016, S. 198).

- Darüber hinaus zeigt dieser kosmopolitische Blick auch ein weiteres, sozusagen gegenteiliges, Defizit auf, den relativen Verlust an Souveränität des Nationalstaats: In Zeiten intensivierter Globalisierung hat die nationale Demokratie immer weniger die Macht, souveräne Entscheidungen zu treffen, da viele Angelegenheiten inzwischen dem Bereich des Politischen entzogen und von mächtigen transnationalen Playern de facto im Alleingang entschieden werden. Die oft beklagte Theatralisierung der Politik - Politik werde uns als emotional rührendes Schauspiel vorgeführt, während die wirklichen Entscheidungen ganz woanders fielen - hat hier ihren Ursprung. Aber dieser innenpolitische Aspekt - so wichtig er ist - ist nur die eine Seite der Medaille. Die andere besteht darin, dass auch wir - die demokratischen westlichen Staaten, die (immer noch) die stärkste Wirtschaftsmacht der Welt darstellen - weltpolitisch sehr undemokratisch agieren und damit nicht nur ihre eigenen Prinzipien verraten, sondern für viele weltpolitischen Konflikte mitverantwortlich sind. Auch hier ist ein Dilemma auszumachen, da supranationale demokratische Mechanismen zwar nötig wären, aber höchstens in Ansätzen existieren. Und die Forderung nach global citizenship bzw. danach, sich als global citizen zu verhalten, ist ebenfalls der Hinweis auf dieses Dilemma.

GCED macht die Notwendigkeit sichtbar, transnationale Formen der Demokratie als Gegengewicht zu entwickeln, die vor allem den Einfluss all derer stärken, die bei der herrschenden internationalen Anarchie zu kurz kommen. Inzwischen gibt es bereits eine ganze Reihe länderübergreifender Institutionen, internationaler Regime und Dokumente globalen Rechts sowie eine sich globalisierende Zivilgesellschaft, die aber noch viel zu schwach sind, um wirksam gegenzusteuern. Seyla Benhabib drückt hier wohl einen Konsens zwischen kosmopolitischen und postkolonialen Strömungen aus, wenn sie postuliert, man müsse „die Dokumente globalen öffentlichen Rechts und die Rechtsfortschritte in Menschenrechtsabkommen ohne allzu viel utopisches Getöse nüchtern nutzen, um den Aufbau gegenhegemonialer transnati- onaler Bewegungen zu unterstützen, die [...] Rechte grenzüberschreitend einfordern, und um die Neuerfindung und auch Wiedererlangung wertvoller Normen zu ermöglichen [...] (Benhabib, 2016, S. 41).

Fassen wir zusammen: Warum ist GCED für die Bildung zur Demokratie unverzichtbar? Das normative Argument dazu wurde soeben geliefert: weil es heute keine genuine national abgeschottete Demokratie mehr geben kann. Sie wäre nicht wirklich demokratisch. GCED verweist auf die Notwendigkeit transnationaler Demokratie - schon allein dadurch, dass sie uns dazu bringt, uns mit der eigenen „imperialen Lebensweise“ (Brand, 2017) auseinanderzusetzen - eine Voraussetzung dafür, die Demokratiedefizite im Weltmaßstab und ihre Gründe angemessen zu verstehen.

Das pädagogische Argument wiederum lautet: Demokratiebildung, ausschließlich im nationalen Paradigma gedacht, kann gar nicht mehr gelingen, weil sie von den Lernenden nicht angenommen wird. Angesichts der multikulturell zusammengesetzten Wohnbevölkerung sitzen heute diejenigen, die alle Rechte haben, in derselben Klasse wie die demokratischen Habenichtse. Die Schule ist somit - zumindest im Prinzip - der Ort des Zusammentreffens der Privilegierten mit denjenigen, die aufgrund der Herkunft ihrer Familien vielleicht stärker für Unrecht in anderen Erdteilen sensibel sind, das auch unter „unserer“ Mithilfe entstanden und aufrechterhalten wird. Wie kann da Bildung zur Demokratie den Vorwurf ignorieren, es sei gar nicht vorgesehen, dass all diejenigen, die laut Lehrplan zur Demokratie gebildet werden sollen, überhaupt in den Genuss aller demokratischen Rechte kommen?

Allerdings wird dieses Zusammentreffen der Verschiedenen, vor allem in Ländern wie Deutschland und Österreich, in denen die Gesamtschule kein Regelfall ist, durch vielfältige Segregationsmaßnahmen verhindert. Vor allem in den höheren Schulen sind die einheimischen Schüler/-innen massiv überrepräsentiert. Soziale Unterschiede und solche der Herkunft werden, als Begabungsunterschiede interpretiert, durch getrennte Schulsysteme zementiert bzw. weichen einheimische Schüler/-innen oft vor "zu viel Mischung“ in andere Schulbezirke oder in Privatschulen aus (Herzog-Punzenberger, 2017; Sachverständigenrat deutscher Stiftungen für Integration und Migration, 2013).

Somit kommt noch ein drittes, bildungspolitisches Argument für die Notwendigkeit von GCED hinzu. Sie macht auf strukturell verursachte Ungleichheiten im Bildungssystem aufmerksam und stellt zugleich den Zusammenhang zu den demokratiepolitischen Konsequenzen aus einer nationalstaatlichen Innen- und einer Außensicht her. Diese wird heute umso wichtiger, je mehr rechtsnationale Regierungen in Europa zunehmen. Denn während liberale Regierungen Ungleichheit beklagen und von der Schule ihren Beitrag zu Chancengerechtigkeit und sozialem Aufstieg einfordern, ohne sich zu strukturellen Reformen zu entschließen, die das erst ermöglichen würden, sind inzwischen immer mehr rechte Regierungen an der Macht, die sich sogar von der Idee der Chancengerechtigkeit zu verabschieden scheinen und problematische bildungspolitische Weichen stellen, die Ungleichheiten noch zu verstärken drohen - wie die Auseinandersetzung um segregierte ,Deutsch-Förderklassen ' in Österreich 2018 zeigt. $^{2}$

\section{Dekolonialisierte Politische Bildung}

Verzichtet Politische Bildung auf eine kosmopolitisch-dekoloniale Perspektive, wie sie hier angedeutet wurde, so entsteht eine 
argumentative Engführung. Die praktischen pädagogischen Folgen dieser Verkürzung sind immer wieder kritisiert worden (Andreotti, 2006; Danielzik et al., 2013; Marvakis, 2013). Dabei geht es v.a. um folgende Kritikpunkte:

- Diese Pädagogik ist hilflos gegenüber rechten europäisch-rassistischen Ideologien, da sie nicht beachtet, dass diese Ideologien auch an rebellische Elemente und das Streben nach Würde von Jugendlichen appellieren.

- Sie kann einem antikolonial argumentierenden, muslimisch unterfütterten Extremismus zu wenig entgegensetzen, da sie dessen antikoloniale Impulse nicht produktiv aufnehmen kann.

- Sie macht sich, mangels einer Alternative, zur Unterstützerin eines (neo-)liberalen Demokratiemodells, das in der westlichen Demokratie die höchste Form gesellschaftlichen Zusammenlebens sieht, der alle anderen Nationen nachzueifern hätten.

- Sie kann die Menschenrechte (z.B. Rechte von Frauen) nicht wirksam genug verteidigen, solange sie nicht zugleich auch die Verletzung von Menschenrechten anklagt, die Demokratien gegenüber Migrant/inn/en oder Ländern des Globalen Südens begehen.

- Sie kann weltweit bestehende Ungleichheiten und Ungerechtigkeiten zwar ansprechen und kritisieren, verfällt aber in Patronizing als Haltung gegenüber den, weniger entwickelten' Nationen; denn sie sieht nur in Entwicklungshilfe und ,Bewusstseinsbildung im Norden entscheidende Faktoren für Veränderungen, ohne die ,imperiale Lebensweise infrage zu stellen.

- Sie verzichtet weitgehend darauf, die vielfältigen, allerdings keineswegs immer, westlich-demokratischen' Formen des Widerstands politischer Bewegungen im Süden zu lehren und erweckt damit den Eindruck, es gäbe keine politischen Handlungsmöglichkeiten.

Eine Politische Bildung, die den GCED-Ansatz integriert hat, ist auch in ,demokratischen Staaten' bis zu einem gewissen Grade ein ,subversives' Unternehmen. Denn sie beschränkt sich nicht darauf, Lernenden demokratische Spielräume aufzuzeigen und sie zu ermuntern, diese auch zu nutzen. Sie leitet Lernende darüber hinaus an, Grenzen und Defizite der Demokratie auszuloten sowie den Ideologien entgegenzutreten, die demokratieverhindernd sind. Dies ist einerseits der Rassismus, der kategoriale Unterschiede von Menschen herstellt, und damit die Idee der Gleichwertigkeit unterminiert. Es ist andererseits jede Form des universalistischen Moralismus, der eine gönnerhafte Haltung gegenüber dem globalen Süden an den Tag legt und gleichzeitig die strukturellen Herrschaftsverhältnisse einer ungerechten Weltordnung nicht antastet. Eine Politische Bildung im GCED Paradigma bietet hingegen Lernenden analytische Instrumente, sich selbst anhand ausgewählter Beispiele mit globalen Fragen bzw. (auch) globalen Aspekten lokal aktueller Themen zu beschäftigen und sich damit jenes Wissen anzueignen, das erst die Ausübung demokratischer Rechte ermöglicht. Denn wir dürfen das Globale nicht im ,Anderswo' suchen, sondern im Hier und Jetzt: GCED „encourages students to adopt a critical understanding of globalization, to reflect on how they and their nations are implicated in local and global problems and to engage in intercultural perspectives“ (Pashby, 2012, S. 9).
Ohne eine dekolonial-kosmopolitische Perspektive ist eine echte Bildung zur Demokratie heute nicht mehr zu haben.

\section{Anmerkungen}

1 Vgl. dazu etwa Benjamin Masse-Stamberger: «Fin du monde» contre «fin du mois», la rhétorique méprisante de nos élites. In Marianne, 29/11/2018. https://www. marianne.net/debattons/billets/fin-du-monde-contre-fin-du-mois-la-rhetorique-meprisante-de-nos-elites (Zugriff am 31.12.2018) sowie das Dossier «Le soulèvement français» in Le Monde Diplomatique, Janvier 2019, 1 et 13 à 19.

2 Vergleiche dazu: https://derstandard.at/2000084490603/Deutschfoerderklassen -eher-segregierend

\section{Literatur}

Andreotti, V. (2006). Soft versus critical global citizenship education. Development Education. Policy \& Practice - A Development Education Review, 3, 40-51.

Benhabib, S. (2016). Kosmopolitismus ohne Illusionen. Menschenrechte in unruhigen Zeiten. Frankfurt: Suhrkamp.

Bobbio, N. (1985). Die Zukunft der Demokratie. PROKLA. Zeitschrift Für Kritische Sozialwissenschaft, 15(61), 23-40. https://doi.org/10.32387/prokla.v15i61.1390

Brand, U. \& Wissen, M. (2017). Imperiale Lebensweise. Zur Ausbeutung von Mensch und Natur in Zeiten des globalen Kapitalismus. München: oekom.

Césaire, A. (1968). Über den Kolonialismus. Berlin: Wagenbach.

Danielzik, C.-M., Kiesel, T. \& Bendix, D. (2013). Bildung für nachhaltige Ungleichheit? Eine Postkoloniale Analyse von Materialien der Entwicklungspolitischen Bildungsarbeit in Deutschland. Berlin: glokal e. V.

Fraser, N. (2017). Vom Regen des progressiven Neoliberalismus in die Traufe des reaktionären Populismus. In H. Geiselberger (Hrsg.), Die große Regression. Eine internationale Debatte über die geistige Situation der Zeit (S. 77-92). Frankfurt: Suhrkamp.

Geiselberger, H. (Hrsg.). (2017). Die große Regression. Eine internationale Debatte über die geistige Situation der Zeit. Frankfurt: Suhrkamp.

Go, J. (2016). Postcolonial thought and social theory. Oxford: University Press. doi. org/10.1093/acprof:oso/9780190625139.001.0001

Grobbauer, H. \& Wintersteiner, W. (2016). Global Citizenship als Bildungsstrategie. Erfahrungen mit dem Universitätslehrgang „Global Citizenship Education“. Bildung und Erziehung 69(3), 325-340. doi.org/10.7788/bue-2016-0306

Henkenborg, P. (2009). Demokratie-Lernen - eine Philosophie der Politischen Bildung. Österreichische Zeitschrift für Politikwissenschaft (ÖZP), 38(3), 277-291.

Herzog-Punzenberger, B. (2017). Segregation - oder die Vielfalt in den Schulklassen? POLICY BRIEF \#05. Wien: Kammer für Arbeiter und Angestellte. https://www.arbei terkammer.at/infopool/wien/PB05_Segregation.pdf

Marvakis A. (2013). Zur Dialektik des neuen Faschismus (nicht nur) in Griechenland - oder: "Der Mensch lebt nicht vom Brot allein, besonders wenn er keines hat». In L. Billmann \& J. Held (Hrsg.), Solidarität in der Krise (S. 281-296). Wiesbaden: Springer. doi.org/10.1007/978-3-658-00912-0_14

Mason, P. (2017). Keine Angst vor der Freiheit. In H. Geiselberger (Hrsg.), Die große Regression. Eine internationale Debatte über die geistige Situation der Zeit (S. 149-174). Frankfurt: Suhrkamp.

Mignolo, W. D. (2011). The Darker Side Of Western Modernity. Global Futures, Decolonial Options. Durham: Duke University Press. doi.org/10.1215/9780822394501

Pashby, K. (2012). Questions for Global Citizenship Education in the Context of the 'New Imperialism'. For Whom, by Whom? In V. de Oliveira Andreotti \& L. M. T.M. de Souza (Hrsg.), Postcolonial Perspectives on Global Citizenship Education (S. 9-26). New York: Routledge.

Rancière, J. (2008). Zehn Thesen zur Politik. Zürich-Berlin: diaphanes.

Sachverständigenrat deutscher Stiftungen für Integration und Migration (2013). Segregation an deutschen Schulen. Ausmaß, Folgen und Handlungsempfehlungen für bessere Bildungschancen. Berlin. https://www.stiftung-mercator.de/media/downloads/3_Publikationen/SVR_Studie_Bildungssegregation_Juli_2013.pdf

Sander, W. (2018). Bildung. Ein kulturelles Erbe für die Weltgesellschaft. Frankfurt: Wochenschau.

\section{Dr. Werner Wintersteiner}

ist Univ.-Prof. i. R., Gründer und ehemaliger Leiter des Zentrums für Friedensforschung und Friedensbildung der Universität Klagenfurt; Wissenschaftlicher Leiter des Master Lehrgangs "Global Citizenship Education". Arbeitsschwerpunkte: Friedenspädagogik und Global Citizenship Education; Literatur, Politik und Frieden; (transkulturelle) literarische Bildung; Kulturwissenschaftliche Friedensforschung; Schwerpunkt Alpen-Adria-Region. 